\title{
Graphic Sequences with a Realization Containing a Friendship Graph
}

\author{
Michael J. Ferrara* \\ Department of Mathematics \\ University of Colorado at Denver \\ Ronald J. Gould \\ Department of Mathematics and Computer Science \\ Emory University \\ John R. Schmitt \\ Department of Mathematics \\ Middlebury College
}

November 10, 2005

\begin{abstract}
For any simple graph $\mathrm{H}$, let $\sigma(H, n)$ be the minimum $m$ so that for any realizable degree sequence $\pi=\left(d_{1}, d_{2}, \ldots, d_{n}\right)$ with sum of degrees at least $m$, there exists an $n$-vertex graph $G$ witnessing $\pi$ that contains $H$ as a weak subgraph. Let $F_{k}$ denote the friendship graph on $2 k+1$ vertices, that is, the graph of $k$ triangles intersecting in a single vertex. In this paper, for $n$ sufficiently large, $\sigma\left(F_{k}, n\right)$ is determine precisely.

Keywords: degree sequence, potentially graphic sequence, friendship graph.
\end{abstract}

\section{Introduction}

Let $G$ be a simple undirected graph, without loops or multiple edges. Let $V(G)$ and $E(G)$ denote the vertex set and edge set of $G$ respectively. For a

*mferrara@math.cudenver.edu 
vertex $v \in V(G)$, let $N(v)$ denote the set of neighbors (or neighborhood) of $v$, and $d(v)$ the degree of $v$, that is the order of $N(v)$. We let $\bar{G}$ denote the complement of $G$. Denote the complete graph on $t$ vertices by $K_{t}$, and the friendship graph by $F_{k}$, where $F_{k}$ is the graph of $k$ triangles intersecting in a single vertex.

A sequence of nonincreasing, nonnegative integers

$$
\pi=\left(d_{1}, d_{2}, \ldots, d_{n}\right)
$$

is called graphic if there is a (simple) graph $G$ of order $n$ having degree sequence $\pi$. In this case, $G$ is said to realize $\pi$, and we will write $\pi=\pi(G)$. If a sequence $\pi$ consists of the terms $d_{1}, \ldots, d_{t}$ having multiplicities $m_{1}, \ldots, m_{t}$, we may write $\pi=\left(d_{1}^{m_{1}}, \ldots, d_{t}^{m_{t}}\right)$. There are numerous elementary methods to check if a given sequence is graphic (for example, see $[3,7,8]$ ).

Define $\sigma(H, n)$ to be the smallest integer $m$ so that for every $n$-term graphic degree sequence with degree sum at least $m$ there exists a realization containing $H$ as a weak subgraph. Such sequences are said to be potentially $H$-graphic. Note that in the definition of this function one only needs to replace the quantifier 'there exists a' with 'for every' to obtain a value that is two more than twice the Turán number, ex $(n, H)$. In this paper we determine the value of $\sigma\left(F_{k}, n\right)$.

For a survey of similar results we refer the reader to [18], and for any undefined terms to [1]

\section{Useful Known Results}

In [4] Erdős, Jacobson and Lehel conjectured that

$$
\sigma\left(K_{t}, n\right)=(t-2)(2 n-t+1)+2 .
$$

The conjecture rises from consideration of the graph $K_{(t-2)}+\bar{K}_{(n-t+2)}$, where + denotes the join. It is easy to observe that this graph contains no $K_{t}$, is the unique realization of the sequence

$$
\left((n-1)^{t-2},(t-2)^{n-t+2}\right),
$$

and has degree sum $(t-2)(2 n-t+1)$. Erdös et al. proved the conjecture for $t=3$ and $n \geq 6$. The cases $t=4$ and 5 were proved separately (see [6] and [10], and [11]). For $t \geq 6$ and $n \geq\left(\begin{array}{l}t \\ 2\end{array}\right)+3$, Li, Song \& Luo [12] proved the conjecture true via linear algebraic techniques. Later, the present authors 
proved all cases of the conjecture via induction on $t$ using graph theoretic techniques [5].

The following summarizes these results.

Theorem 1 For $t \geq 3$ and $n>n_{0}(t)$,

$$
\sigma\left(K_{t}, n\right)=(t-2)(2 n-t+1)+2 .
$$

The following results will be used in the proof of our main result.

Theorem 2 (Erdős-Gallai [3]) A nonincreasing sequence of nonnegative integers

$$
\pi=\left(d_{1}, d_{2}, \ldots, d_{n}\right)
$$

$(n \geq 2)$ is graphic if, and only if, the sum of the degrees is even and for each integer $k, 1 \leq k \leq n-1$,

$$
\sum_{i=1}^{k} d_{i} \leq k(k-1)+\sum_{i=k+1}^{n} \min \left\{k, d_{i}\right\} .
$$

The following is an extension of a theorem of Rao [17].

Theorem 3 ([6]) If $\pi$ is a graphic sequence with a realization $G$ containing $H$ as a subgraph, then there is a realization $G^{\prime}$ of $\pi$ containing $H$ with the vertices of $H$ having the $|V(H)|$ largest degrees of $\pi$.

Theorem 4 ([13], [14]) Let $\pi=\left(d_{1}, d_{2}, \ldots d_{n}\right)$ be a non-increasing sequence of non-negative integers, where $d_{1}=m$ and the degree sum is even. If there exists an integer $n_{1} \leq n$ such that $d_{n_{1}} \geq h \geq 1$ and $n_{1} \geq \frac{1}{h}\left[\frac{(m+h+1)^{2}}{4}\right]$, then $\pi$ is graphic.

Theorem 5 ([15]) Let $n \geq 2 r+2$ and $\pi=\left(d_{1}, d_{2}, \ldots d_{n}\right)$ be graphic with $d_{r+1} \geq r$. If $d_{2 r+2} \geq r-1$, then $\pi$ is potentially $K_{r+1}$-graphic.

The value of $\sigma\left(k K_{2}, n\right)$ was determined in [6].

Theorem 6 ([6]) $\sigma\left(k K_{2}, n\right)=(k-1)(2 n-k)+2$.

The lower bound for $\sigma\left(k K_{2}, n\right)$ is easy to obtain by considering the graph $G^{\prime}=K_{k-1}+\bar{K}_{n-k+1}$. This graph is the unique realization of the degree sequence $\pi=\left((n-1)^{k-1},(k-1)^{n-k+1}\right)$, contains no matching of size $k$, and has degree sum $(k-1)(2 n-k)$. 


\section{The Main Theorem}

Erdös et al. [2], showed that any graph on $n$ vertices having at least

$$
\left\lfloor\frac{n^{2}}{4}\right\rfloor+ \begin{cases}k^{2}-k+1 & \text { if } k \text { is odd } \\ k^{2}-\frac{3}{2} k+1 & \text { if } k \text { is even }\end{cases}
$$

edges contains a copy of $F_{k}$. The following is an analogue to this result. Our proof utilizes a technique developed in [16].

Theorem 7 For $k \geq 1$ and $n \geq \frac{9}{2} k^{2}+\frac{7}{2} k-\frac{1}{2}$,

$$
\sigma\left(F_{k}, n\right)=k(2 n-k-1)+2 \text {. }
$$

As $F_{1}$ is isomorphic to $K_{3},(1)$ is established for $k=1$ by Theorem 1 . Equation (1) was established for $k=2$ by Lai in [9]. Our proof of Theorem 7 holds for all $k \geq 1$.

Proof: To see that $\sigma\left(F_{k}, n\right) \geq k(2 n-k-1)+2$, consider the graph $G=K_{1}+G^{\prime}$, where $G^{\prime}$ is any graph on $n-1$ vertices where no realization of the degree sequence given by $G^{\prime}$ contains $k$ disjoint edges. We may choose $G^{\prime}$ to be the graph $K_{k-1}+\bar{K}_{n-k}$ as in Theorem 6 . Thus $G$ is the graph $K_{k}+\bar{K}_{n-k}$. The graph $G$ is the unique realization of the degree sequence $\pi=\left((n-1)^{k},(k)^{n-k}\right)$ and has degree sum equal to $k(n-1)+(n-k) k=$ $k(2 n-k-1)$. To see that $G$ contains no copy of $F_{k}$ first notice that any $k+1$ vertices of $F_{k}$ must contain at least one edge. Now if $G$ were to contain a copy of $F_{k}$ it must contain at least $k+1$ of its vertices from the subgraph $\bar{K}_{n-k}$ of $G$, however this subgraph does not contain an edge. This establishes the lower bound.

We now establish the upper bound through a sequence of lemmas.

The following establishes that there are sufficiently many vertices of sufficiently large degree in any graph with the degree sum at least that given by (1).

Lemma 1 Let $S=\left(d_{1}, \ldots, d_{n}\right)$ be a non-increasing graphic degree sequence with with degree sum at least $k(2 n-k-1)+2$ and $n>k^{2}+k-2$, then $d_{1} \geq 2 k$ and $d_{2 k+1} \geq 2$.

Proof: To see that $d_{1} \geq 2 k$, suppose otherwise, so $S$ contains no term larger than $2 k-1$. Then the degree sum of $S$ is at most $n(2 k-1)$, a contradiction. 
Suppose now that $d_{2 k+1} \leq 1$. Then, by Theorem 2 ,

$$
\begin{aligned}
\sum_{i=1}^{n} d_{i} & =\sum_{i=1}^{2 k} d_{i}+\sum_{i=2 k+1}^{n} d_{i} \\
\leq & (2 k)(2 k-1)+\sum_{i=2 k+1}^{n} \min \left\{2 k, d_{i}\right\}+\sum_{i=2 k+1}^{n} d_{i} \\
& =4 k^{2}-2 k+2 \sum_{i=2 k+1}^{n} 1 \\
& \leq 4 k^{2}-2 k+2(n-2 k) \\
& =2 n+4 k^{2}-6 k
\end{aligned}
$$

This is a contradiction.

Let $\pi=\left(d_{1}, \ldots, d_{n}\right)$ be a non-increasing, $n$-term graphic sequence with degree sum at least $k(2 n-k-1)+2$. We will now recursively define a sequence $\pi_{1}, \ldots, \pi_{2 k+1}$ of degree sequences. We begin by constructing the sequence $\pi_{1}^{\prime}$, on $n-1$ terms, by deleting $d_{1}$ from $\pi$ and subtracting 1 from the first $d_{1}$ remaining terms. That is,

$$
\pi_{1}^{\prime}=\left(d_{2}-1, d_{3}-1, \ldots, d_{d_{1}+1}-1, d_{d_{1}+2}, \ldots, d_{n}\right) .
$$

We then obtain the sequence $\pi_{1}$ from $\pi_{1}^{\prime}$ by subtracting one from each of the first $2 k$ terms in $\pi_{1}^{\prime}$ and arranging the first $2 k$ terms in non-increasing order and then arranging the last $n-2 k-1$ terms in non-increasing order. (As Lemma 1 guarantees that $d_{2 k+1} \geq 2$ we are assured that this step is feasible.) Let

$$
\pi_{1}=\left(d_{2}^{(1)}, d_{3}^{(1)}, \ldots, d_{n}^{(1)}\right) .
$$

For $2 \leq i \leq 2 k+1$, we obtain the sequence

$$
\pi_{i}=\left(d_{i+1}^{(i)}, \ldots, d_{n}^{(i)}\right)
$$

of length $n-i$ from

$$
\pi_{i-1}=\left(d_{i}^{(i-1)}, \ldots, d_{n}^{(i-1)}\right)
$$

by deleting $d_{i}^{(i-1)}$ from $\pi_{i-1}$, subtracting one from the largest $d_{i}^{(i-1)}$ nonnegative remaining terms and arranging the first $2 k+1-i$ terms in nonincreasing order and then arranging the last $n-2 k-1$ terms in nonincreasing order. 
Lemma 2 If $\pi_{2 k+1}$ is graphic then $\pi$ is potentially $F_{k}$-graphic.

Proof: Clearly, if $\pi_{2 k+1}$ is graphic, then $\pi_{1}$ is graphic. As $\pi$ is graphic, the Havel-Hakimi algorithm [7, 8] implies that $\pi_{1}^{\prime}$ is graphic. If we can show that there is a realization of $\pi_{1}^{\prime}$ that has a matching on those vertices of degree $d_{2}-1, \ldots, d_{2 k+1}-1$, then clearly $\pi$ is potentially $F_{k}$-graphic. Let $G_{1}^{\prime}$ be a realization of $\pi_{1}^{\prime}$ and let $G_{1}$ be a realization of $\pi_{1}$ such that $V_{1}=V\left(G_{1}\right)=V\left(G_{1}^{\prime}\right)=\left\{v_{2}, \ldots, v_{n}\right\}$ with $d_{G_{1}}\left(v_{i}\right)=d_{G_{1}^{\prime}}\left(v_{i}\right)-\delta_{i}$ where $\delta_{i}=1$ for $2 \leq i \leq 2 k+1$ and $\delta_{i}=0$ otherwise.

Let $H$ be a copy of $K_{n-1}$ on $V_{1}$, and consider the function $W: E(H) \rightarrow$ $\{-1,0,1\}$ defined by

$$
W\left(v_{i} v_{j}\right)=\left\{\begin{array}{cr}
-1 & v_{i} v_{j} \in E\left(G_{1}\right) \backslash E\left(G_{1}^{\prime}\right) \\
1 & v_{i} v_{j} \in E\left(G_{1}^{\prime}\right) \backslash E\left(G_{1}\right) \\
0 & \text { otherwise. }
\end{array}\right.
$$

The function $W$ induces a weighting $w: V_{1} \rightarrow \mathbb{Z}$, where the weight of a vertex $v$ is the sum of the weights of the edges incident to $v$ in $H$. If we let $X=\left\{v_{2}, \ldots, v_{2 k+1}\right\}$, then one can see that $w(v)=1$ if $v$ is a member of $X$ and $w(v)=0$ otherwise.

It will be shown that there exists a collection of trails $T_{1}, \ldots, T_{k}$ in $H$ that satisfy the following four properties.

(1) $T_{1}, \ldots, T_{k}$ are edge disjoint.

(2) The end-vertices of $T_{1}, \ldots, T_{k}$ are distinct vertices in $X$, and hence cover $X$.

(3) The first edge, and last edge, in each trail has weight 1 under $W$.

(4) If $T_{j}=e_{1} e_{2} \ldots e_{p}$ then $W\left(e_{i+1}\right)=-W\left(e_{i}\right)$ for $1 \leq i \leq p-1$.

If $v$ lies on $T_{i}$, let $w_{i}$ denote the vertex weighting induced by $\left.W\right|_{E\left(T_{i}\right)}$. Note that if $v$ is an end-vertex of $T_{i}$ then $w_{i}(v)=1$ and if $v$ is an internal vertex of $T_{i}$, then $w_{i}(v)=0$.

We begin by showing that $T_{1}$ exists. Select $v_{2}$ as an end-vertex of $T_{1}$. Note that as $v_{2}$ is in $X, w\left(v_{2}\right)=1$ so there is some edge $e$ in $H$ incident to $v_{2}$ with $W(e)=1$. If there is such an edge between $v_{2}$ and some other vertex $x$ in $X$, let $T_{1}$ consist of the edge $v_{2} x$. Otherwise, there is an edge $v_{2} y$ such that $W\left(v_{2} y\right)=1$ and $y$ is not in $X$. Include the edge $v_{2} y$ in $T_{1}$. As $w(y)=0$, there is some edge incident to $y$ having weight -1 , which is then 
included in $T_{1}$. Continue this process, and construct an alternating $+1 /-1$ trail in $H$. If at any point there exists an edge $e$ with $W(e)=1$ satisfying (1) - (4) above then include $e$ in $T_{1}$. As this process clearly terminates, we wish to show that it must terminate with such a choice. Assume not, so that $T_{1}$ is an alternating $+1 /-1$ trail that violates $(2)$ or $(3)$ above. We show that such a trail can be extended. Assume first that (2) is violated. If the end-vertex of this trail is $v_{2}$, then as $w\left(v_{2}\right)=1$, our choice for the initial edge of $T_{1}$ implies that we can clearly continue the trail regardless of the weight of the final edge. If the end-vertex of the trail is some $v$ in $V \backslash X$ then we note that $w(v)=0$, and each time, if any, that $v$ appears previously in the trail, it is adjacent to one edge of weight +1 and one edge of weight -1 . Thus, if the last edge $e$ on the trail has weight $W(e)$ (which is necessarily +1 or -1 ), there is some edge not already in the trail which is adjacent to $v$ and has weight $-W(e)$ and the trail can be extended. If we assume that (2) is satisfied, but (3) is violated then the last vertex on the trail is some $x$ in $X \backslash\left\{v_{2}\right\}$ but the last edge $e$ added to the trail has weight $W(e)=-1$. However, $w(x)=1$, which implies that we can extend the trail. Hence, $T_{1}$ exists.

Assume that trails $T_{1}, \ldots, T_{j}$ exist satisfying (1) - (4) and without loss of generality, let the end vertices of $T_{i}$ be $v_{2 i}, v_{2 i+1}$. Note that if $v$ is in $\left\{v_{2}, \ldots, v_{2 j+1}\right\}$ then

$$
\sum_{i=1}^{j} w_{i}(v)=1
$$

and otherwise,

$$
\sum_{i=1}^{j} w_{i}(v)=0 .
$$

To show trail $T_{j+1}$ exists, begin with $v_{2 j+2}$ as an end-vertex. As $w\left(v_{2 j+2}\right)=$ 1 and

$$
\sum_{i=1}^{j} w_{i}\left(v_{2 j+2}\right)=0,
$$

there is some edge $e$ in $H$ adjacent to $v_{2 j+2}$ with $W(e)=1$ that does not lie in any of $T_{1}, \ldots, T_{j}$. If there is such an edge between $v_{2 j+2}$ and some other vertex $x$ in $X \backslash\left\{v_{2}, \ldots, v_{2 j+2}\right\}$, let $T_{j+1}$ consist of the edge $v_{2 j+2} x$. Otherwise, we will proceed in a manner similar to the construction of $T_{1}$, described above. That is, it can be shown that $T_{j+1}$ is an alternating $+1 /-1$ trail, which is edge disjoint from $T_{1} \ldots, T_{j}$. If at any point $T_{j+1}$ can be extended by an edge $e$ of weight $W(e)=1$ to a vertex in $X \backslash\left\{v_{2}, \ldots, v_{2 j+2}\right\}$ the edge $e$ will be added to $T_{j+1}$. Otherwise, we will assume that $T_{j+1}$ is an alternating trail that violates either (2) or (3). Then, as above, we can use 
the induced weights from the previous trails to extend $T_{j+1}$. As the process of extending $T_{j+1}$ must terminate, we can see that $T_{j+1}$ exists satisfying (1) $-(4)$.

Thus there exists trails $T_{1}, \ldots, T_{k}$ satisfying $(1)-(4)$, and assume without loss of generality that the end-vertices of $T_{i}$ are $v_{2 i}$ and $v_{2 i+1}$ for all $1 \leq i \leq k$. Note that if an edge in $H$ has weight 1 then it is in $G_{1}^{\prime}$ and an edge in $H$ having weight -1 is not in $G_{1}^{\prime}$. For each trail $T_{i}$, if $v_{2 i} v_{2 i+1}$ is an edge in $G_{1}^{\prime}$ do nothing. If $v_{2 i} v_{2 i+1}$ is not an edge in $G_{1}^{\prime}$ add this edge and all edges of weight -1 on $T_{i}$ to $G_{1}^{\prime}$ and remove all edges of weight 1 on $T_{i}$ from $G_{1}^{\prime}$. In the event that $W\left(v_{2 i} v_{2 i+1}\right)=-1$ and $v_{2 i} v_{2 i+1}$ lies in some $T_{j}$, we examine $e_{j}=v_{2 j} v_{2 j+1}$. If $e_{j}$ is in $G_{1}^{\prime}$, then we will proceed as above to add $v_{2 i} v_{2 i+1}$ to $G_{1}^{\prime}$. If $e_{j}$ is not in $G_{1}^{\prime}$, we will add $e_{j}$ to $G_{1}^{\prime}$ and "switch" the edges in $T_{j}$. This will also serve to add the edge $v_{2 i} v_{2 i+1}$ to $G_{1}^{\prime}$. Note that it is not possible for $v_{2 i} v_{2 i+1}$ to lie in some $T_{j}$ with $j \neq i$ if $W\left(v_{2 i} v_{2 i+1}\right)=+1$. Thus we can create a realization of $\pi_{1}^{\prime}$ that contains the matching $v_{2} v_{3}, \ldots, v_{2 k} v_{2 k+1}$, implying that $\pi$ is potentially $F_{k}$-graphic.

Lemma 3 If $n \geq 4 k+2$, and $d_{4 k+2} \geq 2 k-1$ then $\pi$ is potentially $F_{k}$ graphic.

PROOF: If $d_{2 k+1} \geq 2 k$ then $\pi$ is potentially $K_{2 k+1}$-graphic by Theorem 5 , and thus obviously $F_{k}$-graphic.

Otherwise $d_{2 k+1} \leq 2 k-1$, which together with the hypothesis implies that $d_{2 k+1}=d_{2 k+2}=\ldots=d_{4 k+2}=2 k-1$. Thus, for $i=0,1, \ldots, 2 k+1$ the values of $d_{2 k+2}^{(i)}, \ldots, d_{4 k+2}^{(i)}$ differ by at most 1 . Hence $\pi_{2 k+1}$ satisfies, for some $m \geq 1$,

$$
2 k-1 \geq m=d_{2 k+2}^{(2 k+1)} \geq \ldots \geq d_{4 k+2}^{(2 k+1)} \geq m-1 .
$$

If $m=1, \pi_{2 k+1}$ must be graphic as the degree sum of $\pi_{2 k+1}$ is even. If $m \geq 2$, then

$$
\frac{1}{m-1}\left[\frac{(m+(m-1)+1)^{2}}{4}\right] \leq m+2 \leq 2 k+1
$$

By Theorem $4, \pi_{2 k+1}$ is graphic, and hence, by Lemma $2, \pi$ is $F_{k^{-}}$ graphic. 
Lemma 4 Let $\pi$ be an $n$-term graphic degree sequence with $n \geq \frac{9}{2} k^{2}+\frac{7}{2} k-$ $\frac{1}{2}$ and degree sum at least $k(2 n-k-1)+2$. If $d_{4 k+2} \leq 2 k-2$ then $\pi$ is potentially $F_{k}$-graphic.

Proof: First, we claim that $d_{1} \geq 4 k$. If not, then the degree sum of $\pi$ is at most $(4 k-1)(4 k+1)+(n-4 k-1)(2 k-2)$, which is less than $k(2 n-k-1)+2$ for the given values of $n$.

If $d_{1}=n-1$ then the degree sum of $\pi_{1}^{\prime}$ is at least $\sigma\left(k K_{2}, n-1\right)$. Therefore, there exists a realization of $\pi_{1}^{\prime}$ that contains a copy of $k K_{2}$ and thus a realization of $\pi$ that contains a copy of $F_{k}$.

Now suppose there exists an $r$ such that $2 k+1 \leq r \leq d_{1}+1$ such that $d_{r+1}<d_{r}$. As the degree sum of $\left(\pi_{1}^{\prime}\right)$ is at least $\sigma\left(k K_{2}, n-1\right)$ there exists a graph realizing $\pi_{1}^{\prime}$ that contains a copy of $k K_{2}$. Furthermore, by Theorem 3 there exists a realization of $\pi_{1}^{\prime}$ with $k K_{2}$ on those vertices having degree $d_{2}-1, \ldots d_{2 k+1}-1$. This implies that $\pi$ is potentially $F_{k}$-graphic. $d_{d_{1}+2}$.

Otherwise, $n-2 \geq d_{1} \geq d_{2} \geq \ldots \geq d_{2 k+1}=d_{2 k+2}=\ldots d_{4 k+2}=\ldots=$

We may conclude that there exists an $m$ such that

$$
2 k-2 \geq m=d_{2 k+2}^{(2 k+1)} \geq \ldots \geq d_{4 k+2}^{(2 k+1)} \geq m-1 .
$$

We may then complete the proof as in the previous lemma.

Together, Lemma 3 and Lemma 4 imply that $\sigma\left(F_{k}, n\right) \leq k(2 n-k-1)+2$, completing the proof of Theorem 7 .

Acknowledgements: The authors wish to thank the anonymous referee for his many useful comments, which improved the clarity of our work.

\section{References}

[1] Bollobás, B., Extremal Graph Theory, Academic Press Inc. (1978).

[2] Erdős, P., Füredi, Z., Gould, R.J., Gunderson, D.S., Extremal Graphs for Intersecting Triangles, J. Combin. Th., Ser. B 64, (1995), 89-100.

[3] Erdős, P. \& Gallai, T., Graphs with prescribed degrees (in Hungarian) Matemoutiki Lapor 11 (1960), 264-274. 
[4] Erdős, P., Jacobson, M.S., Lehel, J., Graphs Realizing the Same Degree Sequence and their Respective Clique Numbers, Graph Theory, Combinatorics and Applications, Vol. I, 1991, ed. Alavi, Chartrand, Oellerman and Schwenk, 439-449.

[5] Ferrara, M., Gould, R., Schmitt, J., Potentially $K_{s}^{t}$-graphic degree sequences, submitted.

[6] Gould, R.J., Jacobson, M.S., Lehel, J., Potentially G-graphic degree sequences, Combinatorics, Graph Theory, and Algorithms (eds. Alavi, Lick and Schwenk), Vol. I, New York: Wiley \& Sons, Inc., 1999, 387400 .

[7] Hakimi, S.L., On the realizability of a set of integers as degrees of vertices of a graph, J. SIAM Appl. Math, 10 (1962), 496-506.

[8] Havel, V., A remark on the existence of finite graphs (Czech.), Časopis Pěst. Mat. 80 (1955), 477-480.

[9] Lai, C., An extremal problem on potentially $K_{m}-C_{4}$-graphic sequences, submitted.

[10] Li, J., Song, Z., An extremal problem on the potentially $P_{k}$-graphic sequences, The International Symposium on Combinatorics and Applications, June 28-30, 1996 (W.Y.C. Chen et. al., eds.) Tanjin, Nankai University 1996, 269-276.

[11] Li, J., Song, Z., The smallest degree sum that yields potentially $P_{k}$ graphical sequences, J. Graph Theory 29 (1998), no.2, 63-72.

[12] Li, J., Song, Z., Luo, R., The Erdös-Jacobson-Lehel conjecture on potentially $P_{k}$-graphic sequences is true, Science in China, Ser. A, 41 1998, (5):510-520.

[13] Li, J., Yin, J., The smallest degree sum that yields potentially $K_{r, r^{-}}$ graphic sequences, Science in China, Ser. A, 45 (June 2002),(6):694705 .

[14] Li, J., Yin, J., An extremal problem on potentially $K_{r, s}$-graphic sequences, Discrete Math., 260 (2003), 295-305.

[15] Li, J., Yin, J., Two sufficient conditions for a graphic sequence to have a realization with prescribed clique size, submitted.

[16] Yin, J., Chen, G., On Potentially $K_{r_{1}, r_{2}, \ldots, r_{m}}$-graphic Sequences, preprint. 
[17] Rao, A.R., The clique number of a graph with a given degree sequence, Proc. Symposium on Graph Theory (A.R. Rao ed.), MacMillan and Co. India Ltd., Indian Statistical Institute Lecture Notes Series 4 (1979), 251-267.

[18] Rao, S.B., A survey of the theory of potentially $P$-graphic and forcibly $P$-graphic degree sequences, Lecture Notes in Math., No. 855, Springer Verlag, 1981, 417-440. 